\section{Thymectomy in a Patient with Myasthenia Gravis and Crohn's Disease - Anaesthetic Challenges}

\section{Rakesh Garg* and Neha Hasija}

Department of Anesthesiology and Intensive Care, Dr. BRAIRCH, All India Institute of Medical Sciences, Ansari Nagar, New Delhi, India

\section{Introduction}

Crohn's Disease (CD) is a chronic, nonspecific, transmural inflammation of the intestine which may be seen throughout the gastrointestinal tract, from the oropharynx to the anus. Crohn's disease also manifests itself in many extraintestinal symptoms including eyes, skin, and joints [1]. On the other hand, Myasthenia Gravis (MG) is a chronic autoimmune neuromuscular disease which manifests as weakness of the skeletal muscles which further increases with activity. The muscular weakness is prominent with eye and eyelid movement, facial expression, chewing, talking and swallowing. However other muscles group like that involved in breathing and limb movement may also be involved. The etiology for concurrence of both these disease simultaneously in not well elucidated. However, it appears to be autoimmune in nature. The combinations of Myasthenia gravis and Crohn's disease have many challenges in the perioperative period for anaesthesiologist. These challenges are related to anaesthetic technique and anaesthetic drugs interactions during perioperative outcome. The concerns are also related to manifestation of these diseases including body system involvement. We report here successful management of aesthesia challenges posed by a combination of Myasthenia gravis and Crohn's disease in a patient for thymectomy.

\section{Case Report}

A sixty-two year male weighing $50 \mathrm{~kg}$ (BMI $25.5 \mathrm{~kg} / \mathrm{m}^{2}$ ) with a diagnosis of MG and $\mathrm{CD}$ was posted for trans-sternal thymectomy. On reviewing the history, he was diagnosed with $\mathrm{CD}$ five years back and was presently on oral prednisolone $(10 \mathrm{mg})$ once an alternate day and oral mesalazine $(500 \mathrm{mg}$ ) thrice a day. He had intermittent exacerbations and was managed with steroid and immunosuppressant. Presently the disease was under remission with ongoing steroid and mesalazine. Two months back, he was diagnosed to have MG and corresponded to Ossermans classification grade II, i.e., mild generalised weakness associated with ocular symptoms (diplopia). The myasthenic symptoms were optimised with oral pyridostigmine $(60 \mathrm{mg})$ thrice a day and azathioprine $(50 \mathrm{mg})$ once a day.

Presently on examination, patient had mild dyspnoea in supine rather than left or right lateral position. His pulse rate was $60 /$ minute and regular and the blood pressure 134/84 $\mathrm{mmHg}$. On auscultation, chest was bilaterally clear with air entry more towards the right than left. Mouth opening was three fingers and modified Mallampati grade of II, short neck, and submental fat was present. Breath holding time was more than 35 seconds. Ptosis of right eye was present. Preoperative investigations were haemoglobin $13 \mathrm{gm} / \mathrm{dL}$, WBC $9500 / \mathrm{mm}^{3}$, Platelets $2.13 \mathrm{lacs} / \mathrm{mm}^{3}$, random blood sugar $81 \mathrm{mg} / \mathrm{dL}$, blood urea $34 \mathrm{mg} / \mathrm{dL}$, serum creatinine $1.15 \mathrm{mg} / \mathrm{dL}$. Serum sodium $141 \mathrm{meq} / \mathrm{L}, \mathrm{S}$. potassium $3.7 \mathrm{meq} / \mathrm{L}$. Thyroid function tests were within normal limits. Pulmonary function tests revealed mild restriction with a FVC of $70 \%, \mathrm{FEV} 1$ of $62 \%$ and FEV1/FVC of $94 \%$ of predicted values. Chest X-ray revealed mediastinal widening and marked tracheal deviation to right. Elelctrocardiogram (ECG) indicated sinus bradycardia (heart rate of $56 / \mathrm{min}$ ). Ejection fraction

Citation: Garg R, Hasija N (2015) Thymectomy in a Patient with 2: 007.

Received: March 24, 2015; Accepted: July 04, 2015; Published: July 13, 2015 
was $60 \%$ on echocardiography. MRI of chest revealed a well defined lobulated heterogeneous soft tissue density mass $7.3 \times 7.5 \mathrm{~cm}$ in anterior mediastinum extending posteriorly and superiorly with multiple specs of calcification noted within it. Mass was displacing trachea to right and extending to neck on the left side. The large solid mass in anterior mediastinum with infiltration was displacing branches of aorta up to sternum and upper dorsal vertebrae; trachea is pushed to right though normal in calibre. Left brachiocephalic vein was compressed. Multiple lymph nodes were seen in periphery of mass of which few are adherent. AchR Ab were positive $13.46 \mathrm{nmol} / \mathrm{l}(>0.4 \mathrm{nmol} / \mathrm{L}$ - positive). On bronchoscopy, trachea was deviated to right and bilateral vocal cords were normal.

Informed written consent for surgery and anaesthesia including epidural and central line were taken. Patient was kept nil per oral for 6 hours. Premedication included oral ranitidine $(150 \mathrm{mg})$ and oral alprazolam $(0.25 \mathrm{mg})$ in the night before surgery. Morning dose of pyridostigmine was given with sip of water. In the operating room, routine monitors (5 lead electrocardiogram, non-invasive blood pressure, pulse oximeter) were attached and $18 \mathrm{G}$ intravenous access was gained in left forearm. Intravenous hydrocortisone $100 \mathrm{mg}$ was administered. Epidural catheter was placed in T10-11 interspinous space in left lateral position. Patient was turned to right lateral position. Anaesthesia was induced with intravenous fentanyl $(100 \mu \mathrm{g})$ and propofol $(100 \mathrm{mg})$ in incremental doses. Mask ventilation was ensured with sevoflurane in $100 \%$ oxygen. Laryngoscopy with glidescope revealed a Cormack Lehane Grade IIa and $7.5 \mathrm{~mm}$ ID cuffed flexometallic tube with stylet was passed and secured at $21 \mathrm{~cm}$ mark. Injection atracurium $30 \mathrm{mg}$ was given intravenously. Patient's lungs were ventilated on pressure controlled mode with a pressure support of $15-20 \mathrm{~cm}$ of water and Positive End-Expiratory Pressure (PEEP) of $5 \mathrm{~cm}$ of water. The frequency of ventilation was titrated to keep the end-tidal carbon dioxide between 35-40 mmHg. Anaesthesia was maintained with oxygen air mixture, sevoflurane (0.5-0.8 MAC) and propofol infusion. Epidural space was loaded with $10 \mathrm{~mL}$ of 0.125 $\%$ bupivacaine with $25 \mu \mathrm{g}$ fentanyl. After sternotomy, thymus was removed and surgical duration was around 4 hours. The oxygen saturation remained more than $98 \%$ throughout the surgery. The heart rate and blood pressure remained within $20 \%$ of baseline throughout surgery. The pyridostigmine was given through Ryles tube. Patient was shifted to ICU for further management and ventilated initially on pressure controlled mode with pressure support of $10-15 \mathrm{~cm}$ of water and Positive End-Expiratory Pressure (PEEP) of $5 \mathrm{~cm}$ of water and gradually weaned to pressure support on return of respiratory efforts. The pressure support was gradually decreased from $15 \mathrm{~cm}$ of water to $5 \mathrm{~cm}$ of water gradually. He was extubated after 2 hours when respiratory efforts were adequate. The postoperative analgesia was provided with epidural morphine $(2 \mathrm{mg})$ diluted in $8 \mathrm{~mL}$ normal saline and administered twice a day and continued for 4 days. Intravenous paracetamol $1 \mathrm{gm}$ was administered thrice a day for 5 days. Postoperative course was uneventful and patient was discharged on $10^{\text {th }}$ postoperative day. Postoperative steroid, pyridostigmine and mesalizine were continued and patient was advised to follow up in oncology and gastroenterology clinics.

\section{Discussion}

MG is associated with other autoimmune disorders like rheumatoid arthritis, sjögren's syndrome, systemic lupus erythematosis, diabetes mellitus, hypothyroidism and thyrotoxicosis commonly [2-4]. CD is a chronic, nonspecific, transmural inflammation of the gastrointestinal tract. However, the occurrence of both these disease is not commonly seen. There are only 3 case reports till date describing the coincidence of the two diseases.

MG has been reported to be associated with both Ulcerative Colitis (UC) and CD [5-8]. Autoimmune disorders, including MG, occur more frequently in UC than in $\mathrm{CD}$. Autoimmune dysregulation is the central defect in both MG and this Inflammatory Bowel Disease (IBD). Both IBD and MG may be associated with an elevated Carcinoembryonic Antigen (CEA) and decreased peripheral lymphocyte counts that subsequently normalise following thymectomy [9]. This indicates beneficial effect of thymemctomy in patient with $\mathrm{CD}$ which also have concomitant MG. The immunological link between MG and IBD is highlighted by two reports of patients undergoing surgical treatment. One report of a patient with both MG and CD documented improvement in perineal and perianal disease following thymectomy for severe uncontrolled MG [7]. Another patient with both MG and UC demonstrated regression of the myasthenia following proctolectomy [10].

Anaesthesia was managed as standard for thymic mass with myasthenia gravis by avoiding neuromuscular blocking agents prior to intubation, intubating in lateral position, using minimal doses of neuromuscular blockers, epidural analgesia to decrease the dose of inhalational agents, avoiding drugs that precipitate myasthenia, continuation of pyridostigmine and planned late extubation.

Drugs used for Crohn's disease like mesalazine can have interactions with azathioprine (used as immunosuppressant for MG) and can increase the effective dose of azathioprine and chances of toxicity of which low blood count and liver toxicity are most worrisome [11]. These concerns play important role in perioperative management as low blood counts increase risk of infection bleeding. Low platelet count also raises concern for central neuraxial block like epidural catheter placement which remains paramount for adequate pain management in such patients. Monitoring renal functions in patients on mesalazine helps monitoring its side effect on kidneys, and Non-Steroidal Anti-Inflammatory Drugs (NSAIDs) should be avoided [12]. Mesalazine associated lung disease is a known entity including bronchiolitis obliterans, Bronchiolitis Obliterans with Organising Pneumonia (BOOP), and interstitial pneumonitis with the most common was being eosinophilic pneumonitis [13]. Mesalazine associated folic acid deficiency can cause anaemia. Given the gastrointestinal side effects and the possibility of aggravating mucosal inflammation, Non-Steroidal Anti-Inflammatory Drugs (NSAID) should be avoided in IBD [14]. The patients undergoing sternotomy may lead to respiratory compromise and increase morbidity because of surgical handling. So these patients require ventilatory support for varying duration in the postoperative period. Our patient required ventilatory support for around 2 hours but that was primarily because of muscle weakness due to MG.

\section{Conclusion}

Myasthenia gravis associated with Crohn's disease is a rare entity. Thymectomy has been reported to improve symptoms of Crohn's disease and should be done on priority basis for such patients. These patients require vigilant management in the perioperative period to avoid any untoward event. Anaesthesia for this combination can be complicated, drug interactions have to be sought for, side effects of the drugs should be known and adjustments for the same should be made when considering the anaesthetic plan. 


\section{References}

1. Welton ML, Shelton AA, Chang GJ, Varma MG (2008) Colon, Rectum, and Anus. In: Surgery: Basic Science and Clinical Evidence. Norton JA, Barie PS, Bollinger RR, Chang AE, Lowry SF, et al., (Eds.). Springer, New York, USA. Pg no: 1011-1110.

2. Téllez-Zenteno JF, Cardenas G, Estañol B, Garcia-Ramos G, Weder-Cisneros N (2004) Associated conditions in myasthenia gravis: response to thymectomy. Eur J Neurol 11: 767-773.

3. Sarkar S, Mandal K (2008) Hypokalemic periodic paralysis accompanied with myasthenia gravis: A Case report. Int J Anesthesiol 16: 2

4. Ratanakorn D, Vejjajiva A (2002) Long-term follow-up of myasthenia gravis patients with hyperthyroidism. Acta Neurol Scand 106: 93-98

5. Martin RW, Shah A (1991) Myasthenia gravis coexistent with Crohn's disease. J Clin Gastroenterol 13: 112-113.

6. Souadijian JV, Enriquez P, Silverstein MN, Pépin JM (1974) The spectrum of disease associated with thymoma. Coincidence or syndrome? Arch Intern Med 134: 374-379

7. Finnie IA, Shields R, Sutton R, Donnelly R, Morris AI (1994) Crohn's disease and myasthenia gravis: a possible role for thymectomy. Gut 35: 278-279.
8. Foroozan R, Sambursky R (2003) Ocular myasthenia gravis and inflammatory bowel disease: a case report and literature review. $\mathrm{Br} \mathrm{J}$ Ophthalmol 87: 1186-1187.

9. Papatestas AE, Kim U, Genkins G, Kornfeld P, Horowitz SH, et al. (1975) The association of carcinoembryonic antigen and peripheral lymphocytes. Surgery 78: 343-348.

10. Gower-Rousseau C, Reumaux D, Bellard M, Delecourt L, Ribet M, et al (1993) Remission of myasthenia gravis after proctocolectomy in a patient with ulcerative colitis. Am J Gastroenterol 88: 1136-1138.

11. Loftus EV Jr, Kane SV, Bjorkman D (2004) Systematic review: short-term adverse effects of 5-aminosalicylic acid agents in the treatment of ulcerative colitis. Aliment Pharmacol Ther 19: 179-189.

12. Mahmud N, O'Toole D, O'Hare N, Freyne PJ, Weir DG, et al. (2002) Evaluation of renal function following treatment with 5 -aminosalicylic acid derivatives in patients with ulcerative colitis. Aliment Pharmacol Ther 16: 207-215.

13. Epler GR, Colby TV, McLoud TC, Carrington CB, Gaensler EA (1985) Bronchiolitis obliterans organizing pneumonia. N Engl J Med 312: 152-158.

14. Ballantyne JC, Mao J (2003) Opioid therapy for chronic pain. N Engl J Med 349: 1943-1953. 